\title{
Revus
}

Journal for Constitutional Theory and Philosophy of Law / Revija za

ustavno teorijo in filozofijo prava

39 | 2019

Revus (2019) 39

\section{Conceptos institucionales: Una crítica a las posiciones reduccionistas e interpretativistas}

Institutional legal concepts. A critique of the reductionist and the interpretativist position

\section{María Cristina Redondo}

\section{(2) OpenEdition Journals}

Edición electrónica

URL: http://journals.openedition.org/revus/5486

DOI: $10.4000 /$ revus.5486

ISSN: $1855-7112$

\section{Editor}

Klub Revus

Referencia electrónica

María Cristina Redondo, « Conceptos institucionales: Una crítica a las posiciones reduccionistas e interpretativistas », Revus [Online], 39 | 2019, Online since 16 December 2019, connection on 01 January 2020. URL : http://journals.openedition.org/revus/5486 ; DOI : 10.4000/revus.5486

Este documento fue generado automáticamente el 2 enero 2020.

All rights reserved 


\section{Conceptos institucionales: Una crítica a las posiciones reduccionistas e interpretativistas}

Institutional legal concepts. A critique of the reductionist and the

interpretativist position

María Cristina Redondo

\section{Introducción}

El presente trabajo propone una reflexión sobre distintos modos de entender aquellos conceptos jurídicos que delimitan, y consecuentemente permiten identificar instituciones jurídicas. Así concebidos, estos conceptos constituyen un subtipo dentro de una clase más amplia de conceptos que designan instituciones sociales en general. ${ }^{1}$ Algunos de estos conceptos son muy específicos e identifican instituciones locales existentes solo en algunos sistemas jurídicos como, por ejemplo, un tipo inusual de contrato o de autoridad administrativa. Otros son más compresivos, identifican instituciones que gozan de una larga tradición y de las cuales encontramos ejemplos en numerosos lugares y momentos históricos. Ejemplos de conceptos de este último tipo serían el concepto de contrato, el de autoridad jurisdiccional, o el caso límite del concepto de derecho en sentido objetivo, en la medida en que designa a un conjunto de normas, i.e. un tipo de institución social. Ciertamente, como se verá, parte del desacuerdo se basa en el distinto modo en que cada posición entiende los conceptos en general. En cualquier caso, la reflexión sobre esta clase de conceptos jurídicos pone un desafío interesante a la teoría del derecho, entre otras razones, porque lo que ellos delimitan, i.e. una clase de institución jurídica, puede considerarse un tipo de 'realidad' social o institucional. Este tipo de realidad, por una parte, tiene como sustrato una práctica de comportamiento susceptible de ser identificada desde un punto de vista empírico, e incluso naturalista. Sin embargo, por otra parte, está dotada de contenido normativo. Es decir, consiste en un conjunto de normas que se resiste a ser reducido a 
un dato empírico. A su vez, el contenido normativo de las instituciones (i.e. de la realidad institucional) se presta a ser identificado y analizado desde distintos puntos de vista. Por ejemplo, desde la perspectiva de la filosofía del lenguaje que tanto ha reflexionado sobre el contenido de las instituciones lingüísticas, pero, sin duda, también desde la perspectiva de la filosofía práctica, ya sea política o moral. Lamentablemente, en el debate sobre la identificación de instituciones jurídicas, estas distintas perspectivas no suelen presentarse como igualmente factibles, sino como enfoques excluyentes entre sí, que proponen el modo correcto de entender, identificar y analizar los conceptos aplicables a tales instituciones.

2 En el presente trabajo me interesa hacer lo siguiente. En la primera parte me referiré a tres propuestas existentes en la teoría jurídica que, en apariencia, constituyen tres diferentes modos de entender e identificar los conceptos jurídicos institucionales. En primer lugar, tomaré en cuenta la concepción interpretativo-justificativa propuesta por la teoría pospositivista de Ronald Dworkin, en segundo lugar, me referiré a la concepción descriptiva y reduccionista asociada a la teoría realista de Alf Ross, y, por último, me detendré en la concepción criterial sostenida por un positivismo jurídico de tipo normativista (en adelante PJN). ${ }^{2}$ Ahora bien, la propuesta metodológica 'analítica' y 'metateórica' asociada al PJN ha sido ampliamente criticada en la filosofía contemporánea. Conforme a ciertos argumentos, ella no debería considerarse una opción disponible, o bien en virtud del grave error en el que estaría basada, o bien en virtud de la escasa fecundidad de la misma. Este trabajo no tiene la ambición de afrontar el debate general sobre la posibilidad de los enunciados analíticos y metateóricos. En modo muy circunscripto, intentaré mostrar dos cosas. Por una parte, en qué sentido el enfoque analítico y metateórico del PJN es posible, i.e. no reducible a, o traducible en, ninguno de los otros dos. Por otra parte, en qué sentido no solo puede ser, sino que de hecho es relevante desde un punto de vista práctico.

En la segunda parte del trabajo aplicaré las tres propuestas previamente identificadas a una institución jurídica concreta. Conforme intentaré mostrar, si las propuestas de Dworkin y Ross constituyen diferentes formas de entender, identificar y analizar un concepto jurídico, cabe concluir que son merecedoras de algunas críticas que, por el contrario, no se aplican a la propuesta del PJN. Sobre esta base, lo que me interesa fundamentalmente es sugerir que las dos primeras propuestas no deberían ser vistas como teorías que compiten entre sí y con el PJN ofreciendo modos incompatibles de entender, identificar y analizar los conceptos jurídicos. En realidad, ellas son contribuciones teóricas complementarias, guiadas por objetivos metodológicos distintos y que responden a preguntas diferentes con relación a las instituciones jurídicas.

\section{Diversas concepciones de los conceptos jurídicos}

\section{1 Los conceptos jurídicos en la perspectiva interpretativista de Dworkin}

4 Ronald Dworkin distingue tres tipos de conceptos: conceptos criteriales (criterial concepts), conceptos de clase natural (natural kind concepts) y conceptos interpretativos (intepretative concepts). ${ }^{3}$ Según este autor, tanto los conceptos criteriales como los de clase natural proporcionan un test para la correcta aplicación de los mismos. En 
contraste, los conceptos interpretativos no ofrecen un test, sino una teoría normativa, siempre controvertida, acerca de cuál es el mejor modo de identificar y justificar aquello a lo que se aplican. ${ }^{4}$ Por lo que se refiere a los conceptos criteriales, Dworkin sostiene que el test que ellos brindan depende de los acuerdos o convenciones existentes en el uso del concepto. Por lo que se refiere a los conceptos de clase natural, como su nombre indica, el test para su correcta aplicación depende de cuál sea la esencia o estructura natural (física o biológica) de los ejemplos o instancias del concepto en cuestión. En todo caso, ninguno de estos dos tipos de conceptos es sensible a los valores que quien los usa atribuye a aquello que los conceptos se aplican, mientras que los conceptos interpretativos sí lo son. Razón por la cual su aplicación es siempre controvertida.

5 Según Dworkin, respecto de una institución como el derecho, por ejemplo, compartimos en alguna medida distintos conceptos: un concepto sociológico, un concepto doctrinal y también uno aspiracional. En cualquier caso, toda teoría jurídica, aun cuando no siempre lo identifique y analice explícitamente, usa un concepto de derecho. En opinión de Dworkin, este concepto es un concepto doctrinal que funciona como un concepto moral, interpretativo. ${ }^{5}$

6 Ahora bien, el concepto doctrinal e interpretativo de derecho - que toda teoría jurídica usa, y respecto del cual se compromete - consiste en un conjunto de principios normativos muy abstractos que, conforme a la opinión expresa o tácita del teórico que lo usa, ofrece la mejor manera de entender y justificar las prácticas y normas concretas en las que el derecho consiste. Como se dijo, este tipo de concepto no proporciona un test o procedimiento para establecer cuáles son los ejemplos a los que se aplica. Es decir, no ofrece un conjunto de criterios decisivos. ${ }^{6}$ El concepto interpretativo de derecho no está determinado por las prácticas lingüísticas de uso, y no puede ser identificado o analizado sin entrar en un debate moralmente comprometido respecto del contenido, siempre controvertido, de la práctica en cuestión. Según Dworkin, frente a casos profundamente injustos, como el del derecho Nazi, podríamos conjeturar que no es posible identificar ninguna teoría justificativa. ${ }^{7}$ Sin embargo, al considerarlo profundamente injusto, cualquiera sea el lenguaje que utilicemos para identificarlo, estaremos tomando una decisión interpretativa y escogiendo entre distintas concepciones controvertidas. ${ }^{8}$ Esta idea confirma una primera tesis general que interesa subrayar aquí: el discurso que identifica una institución normativa (como el derecho en este caso) es siempre interpretativo y se compromete con una tesis moral, i.e. con un conjunto de principios que justifican un modo de entender la institución.

7 Asimismo, según Dworkin, los enunciados teóricos que identifican un concepto doctrinal, interpretativo de derecho no son tesis de carácter semántico, metateórico. En realidad, aunque muy poco 'densas', son tesis del mismo tipo que cualquier otra tesis doctrinal sobre el derecho y se traducen en proposiciones jurídicas concretas aplicables a casos individuales. ${ }^{9}$ Consecuentemente, al tomar posición sobre el concepto tomamos posición acerca de qué tipo de datos o razones fundamentan la verdad de las proposiciones de derecho. Es decir, de las proposiciones que identifican aquello que es obligatorio, prohibido o permitido según un determinado ordenamiento jurídico. ${ }^{10}$ Esta idea confirma una segunda tesis general que interesa subrayar aquí: el discurso que identifica un concepto doctrinal, interpretativo, siempre se compromete con una tesis teórica sustancial sobre cuáles son las condiciones de verdad de las proposiciones que 
identifican aquello que es obligatorio, prohibido o permitido conforme a una determinada institución.

Podría conjeturarse que la propuesta de Dworkin no es relevante respecto de la cuestión que plantea este trabajo. En primer lugar, por ejemplo, podemos entender que, según Dworkin, el único concepto jurídico que funciona como un concepto interpretativo, moral es el concepto general de derecho, y no los conceptos jurídicos más específicos como los de jurisdicción, república, familia, etc. Sin embargo, esta restricción parece implausible ya que el derecho, entendido como una práctica o institución, no parece ser otra cosa que el conjunto de estas otras instituciones y prácticas más circunscriptas, cuya existencia, además, presupone el mismo tipo de actitud interpretativa que subyace al derecho en general. En segundo lugar, podríamos entender que Dworkin no discute sobre el concepto institucional de derecho tal como lo entiendo en este contexto, y que esta última noción corresponde a lo que él denomina un concepto 'sociológico' de derecho. Este concepto sociológico sí es criterial y sobre él la teoría de los conceptos doctrinarios (interpretativos) nada pretende decir. En este sentido, sería un error entender que las tesis de Dworkin sobre el concepto doctrinal e interpretativo de derecho se aplican al concepto que identifica la institución, que sería un concepto sociológico de derecho. Al respecto, las consideraciones que propondré en este trabajo avalan una conclusión en algún modo similar a esta. Es decir, avalan la necesidad de trazar una demarcación entre distintos tipos de aproximaciones teóricas (no tipos de conceptos) que, lejos de ser excluyentes entre sí, son complementarias. Sin embargo, hay dos ideas fuertemente defendidas por Dworkin que sugieren que su propuesta sobre el concepto doctrinal de derecho es vista como competitiva y superadora respecto de la que el 'positivismo analítico' dedica al 'concepto sociológico'.

En efecto, Dworkin propone una división ideal de la teoría jurídica en diferentes etapas.

${ }^{11}$ En una primera etapa o momento semántico, el teórico debería decidir si el concepto de derecho funciona como un concepto criterial (como el de soltería), como uno de clase natural (como el de agua) o como un concepto interpretativo (como el de justicia). En otras palabras, Dworkin aparentemente sostiene que, en una primera fase de la reflexión sobre el derecho, el teórico podría decidir analizar y usar un concepto puramente criterial. ${ }^{12}$ Ahora bien, aunque Dworkin admite explícitamente esta posibilidad, sostiene también dos tesis que la excluyen. En primer lugar, una teoría que identifica y analiza un concepto criterial de derecho, aunque posible, no sería teóricamente interesante. En rigor, según Dworkin, los debates y (aparentes) desacuerdos sobre un concepto criterial ni siquiera plantean una discusión teórica, puesto que requieren solo ponerse de acuerdo sobre una clasificación. Y, ciertamente, si no nos ponemos de acuerdo en la aplicación 'del' concepto criterial, en realidad, o bien estaremos hablando de distintas cosas, o bien estaremos desacordando acerca de cómo deberíamos entenderlas. En segundo lugar, una teoría que sostenga - como sostiene el PJN - que el concepto aplicable a una institución social como el derecho es criterial no solo propone una tesis poco interesante, en realidad, se coloca en una posición imposible. Esto es así porque, según Dworkin, al teorizar sobre el derecho (y cabe recordar que lo mismo sucede respecto de cualquier otra institución normativa) el compromiso con un concepto doctrinal, interpretativo no es opcional o contingente; es ineludible. En otras palabras, la teoría de Dworkin estaría demostrando que es imposible realizar el tipo de indagación que el método analítico del PJN propone, sin comprometerse con un concepto interpretativo. En virtud del carácter social del objeto de estudio, sea o no consciente de ello, toda teoría del derecho asume algún concepto 
interpretativo. Y, en efecto, la entera discusión que Dworkin plantea sobre la propuesta de diversas teorías jurídicas está destinada a mostrar esta conclusión. ${ }^{13}$

No es fácil conjugar las diferentes tesis sostenidas por Dworkin. Tampoco es claro si, y en qué medida, diversos autores que adscriben al PJN han ya aceptado que el concepto de derecho que ellos identifican, efectivamente, implica una teoría doctrinal, interpretativa. De hecho, Dworkin discute con varios de estos autores bajo la hipótesis de que ellos han ya admitido sus críticas fundamentales $y$, consecuentemente, ofrecen un 'positivismo doctrinal'. Es decir, un positivismo cuyas pretendidas tesis semánticas y metateóricas, en realidad, son parte de un debate doctrinal-político-moral que se apoya en un concepto interpretativo de derecho. ${ }^{14}$ En todo caso, no es el objetivo de este trabajo mostrar de qué manera el PJN puede responder o de hecho ha respondido al desafío de Dworkin. Lo que intentaré mostrar mediante el ejemplo que presentaré más adelante es, en primer lugar, que es falsa la tesis según la cual los conceptos que identifican instituciones jurídicas son necesariamente de un único tipo, o bien criteriales, o bien interpretativos. En tal sentido, un debate sobre a cuál de estos dos tipos pertenecen los conceptos que identifican instituciones jurídicas es inconducente puesto que estaría basado en una falsa presuposición. En segundo lugar, es falso que optar por un concepto criterial no permita teorizar en modo interesante o relevante desde un punto de vista sustantivo. En otras palabras, no solo es posible un debate teórico sobre un concepto jurídico sin asumir un compromiso respecto de un concepto interpretativo, sino que los conceptos criteriales que una teoría del derecho puede identificar y el análisis metateórico que puede ofrecer, de hecho, constituyen premisas relevantes al momento de obtener conclusiones sustantivas, ya sean de carácter dogmático o moral.

\section{2 Los conceptos jurídicos en la perspectiva realista de Alf Ross}

11 Alf Ross ha propuesto un célebre análisis del concepto de derecho subjetivo y, en particular, se ha detenido en aquel que delimita la institución de la propiedad. Este tipo de conceptos, que aparentemente se refieren a instituciones jurídicas, en realidad, no designan absolutamente nada. Es decir, expresiones jurídicas como "propietario", "ciudadano", "sociedad anónima" carecerían de toda referencia semántica. ${ }^{15}$

En esta perspectiva, identificar un concepto jurídico institucional es hacer explícito el contenido de las normas jurídicas que lo constituyen. Por ejemplo, la expresión "propietario" no se refiere a algún individuo, propiedad o evento del mundo, sino que sirve para reemplazar, en ciertas ocasiones, una disyunción de hechos condicionantes y, en otras, una conjunción de consecuencias normativas. ${ }^{16}$ Concretamente, cuando decimos: "El propietario tiene permitido percibir los frutos y disponer de los mismos", la palabra "propietario" sustituye y resume la disyunción de hechos que, según un determinado sistema jurídico, condicionan las consecuencias normativas mencionadas (el permiso de percibir y disponer de los frutos). Si no dispusiésemos de esta palabra deberíamos mencionar explícitamente esta disyunción y decir, por ejemplo: "Quien ha recibido una cosa, o por compra, o por herencia, o por prescripción, etc. tiene permitido percibir los frutos y disponer de los mismos". En otras ocasiones, las palabras institucionales sirven para reemplazar el conjunto de consecuencias normativas previstas. Por ejemplo, cuando decimos: "Quien recibe una cosa por compra, herencia o prescripción es el propietario de la misma”. En este caso, lo que hacemos es usar la 
palabra "propietario" en lugar de enumerar la entera lista de obligaciones, prohibiciones y permisos establecidos por el sistema jurídico para este tipo de casos. Es otros términos, decir que alguien es propietario hace posible expresar en términos más sucintos que esa persona ha recibido una cosa por compra, herencia o prescripción y que, como consecuencia, tiene: a) el permiso de percibir los frutos, b) la facultad de transferir la cosa, c) el deber de pagar ciertos impuestos, etc." En general, conforme a Alf Ross:

el concepto no designa fenómeno alguno de ningún tipo que esté insertado entre los hechos condicionantes y las consecuencias condicionadas; únicamente es un medio que hace posible -en forma más o menos precisa- representar el contenido de un conjunto de normas jurídicas. ${ }^{17}$

13 Como podemos observar, los conceptos jurídicos así entendidos no identifican un tipo o clase general de institución (o de entidad, propiedad o hecho institucional), sino instituciones concretas, i.e. conjuntos de normas vigentes en un lugar y tiempo determinados. Los conceptos jurídicos son solo un resumen o una forma breve de presentación de las normas que configuran una determinada institución. A diferencia de lo que propone Dworkin, para Ross los enunciados que identifican un concepto jurídico institucional no implican, sino que son enunciados sobre el contenido normativo de una institución. Es decir, la traducibilidad de los enunciados sobre conceptos jurídicos institucionales en enunciados sobre deberes y derechos concretos es aún más directa que en el caso de Dworkin. Sin embargo, el objetivo del análisis de Ross al mostrar esta traducibilidad puede considerarse casi exactamente opuesto al de Dworkin. Concretamente, este tipo de análisis serviría, en un segundo momento, a mostrar que los enunciados sobre hechos, propiedades $u$ objetos institucionales, en realidad, no pueden ser ni verdaderos ni falsos, puesto que no describen nada, y carecen de 'condiciones de verdad'. Asimismo, el análisis estaría poniendo en evidencia que el lenguaje institucional tiene una función engañosa e ideológica que, lejos de justificar, el teórico - tal como lo hace Ross - debería denunciar. Para este autor, el hecho de que alguien crea en la verdad o falsedad de enunciados sobre la existencia de una realidad o un objeto institucional solo puede explicarse causalmente como resultado o resabio de creencias místicas. En suma, podría decirse que una teoría como la de Ross está intentando mostrar que el lenguaje institucional se apoya en un error sistemático, en creencias falsas en entidades que no existen. Creencias que deberíamos (en el sentido de que tenemos justificación epistémica suficiente para) abandonar. Al respecto, cabe señalar que Alf Ross no propone la eliminación del lenguaje institucional puesto que entiende que es instrumentalmente útil, como vimos, en tanto técnica de presentación. Sin embargo, desde su perspectiva, la ciencia del derecho debería metodológicamente limitarse a describir hechos empíricos y no hechos institucionales. ${ }^{18}$ En esta visión, los hechos, propiedades o entidades institucionales, sencillamente no existen. En tal sentido, intentar describir o seleccionar las características más relevantes de esta aparente 'realidad' institucional es una empresa absurda que se apoya en la falsa presuposición de que hay algo que puede ser objeto de tal descripción o individuación. Analizar un concepto jurídico institucional consiste en mostrar cómo él se reduce a un conjunto de normas existentes en un tiempo y lugar determinados.

Me interesa insistir sobre un punto que considero importante. La propuesta de Ross, a diferencia de la de Dworkin, no hace posible inferir el contenido de una institución a partir del contenido del concepto que se le aplica, sino que directamente hace confluir el contenido del concepto con el contenido de una determinada institución. Esta idea es 
especialmente problemática puesto que conduce a la confusión de dos tipos de normas y de objetos de estudio. Si aceptamos esta equiparación, deviene imposible distinguir entre las reglas semánticas de uso de un concepto y las reglas jurídico-políticas en las que una específica institución consiste. Ahora bien, en un contexto de estudio diferente al jurídico, como el de la zoología, por ejemplo, no se corre el peligro de asimilar, supongamos, los tigres (la realidad objeto de estudio) con el concepto TIGRE, cualquiera sea la concepción que se tenga de este tipo de concepto. En otras palabras, no hay riesgo de confusión entre los enunciados sobre los animales de carne y hueso, ya identificados como tigres conforme a un conjunto de reglas semánticas, y los enunciados sobre el conjunto de criterios o reglas semánticas que seguimos cuando los clasificamos de ese modo. La confusión no es probable porque, entre otras razones, los enunciados en cuestión se refieren a objetos de muy diferente naturaleza, i.e. los tigres no son conjuntos de reglas. Por desgracia, es precisamente esta la primera dificultad que se presenta en los estudios de carácter jurídico. Aquí, el objeto de estudio (el derecho) es de la misma naturaleza que el concepto que permite identificarlo (el concepto de derecho). Dejando de lado las profundas diferencias existentes, ambos son, dependen de, o se expresan en un conjunto de reglas. Ello explica por qué gana plausibilidad la propuesta - que respecto de la realidad natural sería absurda - de que el concepto, o bien implica, o bien se identifica con, el objeto al cual se aplica.

La equiparación de las reglas semánticas que constituyen y gobiernan el uso de un concepto con las reglas jurídico-políticas en las que el objeto consiste es implausible, independientemente del hecho que el estudio de ambos tipos de reglas pueda, o no, reducirse a un estudio de carácter puramente empírico. El primer tipo de reglas establece cómo debemos o cómo es correcto entender ciertos conceptos. El segundo tipo de reglas establece qué está prohibido o permitido decidir o hacer dentro de una comunidad política. Ciertamente, es posible establecer una relación entre estos dos tipos de reglas. Es decir, entre las reglas semánticas que constituyen un concepto jurídico y las reglas jurídico-políticas que constituyen una institución jurídica. En cualquier caso, como veremos más adelante, según la concepción criterial del PJN, esta relación es una de instanciación o ejemplificación: los conjuntos de reglas jurídicopolíticas que conforman cada institución son ejemplos o instancias de aplicación de un concepto jurídico. En contraste, según Dworkin, existe una relación inferencialargumentativa entre el contenido de los conceptos y el de las normas que configuran las instituciones a las que ellos se aplican. Por último, según Ross, se trata de una relación de identidad, puesto que este autor reduce directamente el contenido de los conceptos jurídicos institucionales al contenido de las instituciones jurídicas, respecto del cual tales conceptos son solo un resumen y una técnica de presentación.

Mediante el ejemplo que presentaré en la segunda parte del trabajo intentaré mostrar que la propuesta de Ross es deficitaria respecto de la propuesta del PJN. Esta última, en primer lugar, explica adecuadamente la distinción entre un concepto institucional y las instituciones que lo ejemplifican al caer en su ámbito de aplicación. En segundo lugar, no confunde distintas clases de instituciones: lingüísticas y jurídicas. En tercer lugar, puede distinguir entre hechos institucionales cuya existencia efectivamente se apoya en creencias falsas y aquellos que no. 


\section{3 Los conceptos jurídicos en la perspectiva analítica y metateórica del PJN} jurídicas. Por ejemplo, instituciones jurídicas específicas como el dinero, la monarquía, la propiedad, como así también, la institución jurídica más general que llamamos "derecho" u "ordenamiento jurídico". Un concepto, tal como lo entiende esta posición, es un conjunto de propiedades distintivas que los ejemplos (o al menos los ejemplos paradigmáticos) que recaen en su ámbito de aplicación necesariamente satisfacen. Así entendido, en efecto, un concepto constituye un criterio de identificación de aquello que define. Entre los autores que adhieren al PJN hay discrepancias acerca de si las notas relevantes que configuran un concepto están total o solo parcialmente determinadas por cómo se usan las palabras en una práctica lingüística. Pero, en todo caso, dichas características se consideran relevantes, esenciales, interesantes no en virtud de que así lo acuerdan o creen quienes identifican el concepto, sino en virtud de lo que acuerdan o creen los participantes en la práctica tomada en consideración. Conforme a esta posición, el teórico que identifica un concepto institucional lo hace desde la perspectiva de la tercera persona. Usando un lenguaje usual a partir de la teoría de Hart, cabría decir que, en un sentido práctico, el teórico se coloca en un punto de vista externo, pero toma en cuenta el punto de vista interno de los participantes en la práctica relevante. ejemplo, el concepto de derecho, de propiedad, de matrimonio, etc.) y el contenido de las instituciones que son ejemplos o instancias de aplicación del concepto. El contenido de un concepto jurídico guarda ciertamente una conexión necesaria con la existencia de aquellos que se consideran ejemplos o instancias del mismo. Si decimos que existe un sistema jurídico o que algo es un sistema jurídico estamos aplicando o usando un concepto de sistema jurídico. Sin embargo, y en contraste con quienes sostienen la tesis de la traducibilidad, a partir del contenido de un concepto institucional por sí mismo no es posible inferir conclusiones acerca del contenido normativo de las concretas instituciones que caen bajo su ámbito de aplicación. Y, consecuentemente, tampoco acerca de la justificación de tal contenido. La identificación y el análisis de un concepto jurídico es una empresa que, por hipótesis, se refiere a las condiciones de aplicación del concepto y no ofrece una teoría, ni descriptiva ni justificativa, sobre el contenido normativo de las instituciones que se pueden identificar mediante la aplicación del concepto. Desde este punto de vista, un concepto jurídico es una institución lingüística dotada de un contenido semántico que delimita un tipo de institución jurídica y se refiere a instituciones jurídicas concretas dotadas de contenido jurídico (político o moral).

Esquemáticamente, conforme a esta posición cabe subrayar las siguientes ideas:

(i) La distinción entre la existencia y el contenido de instituciones lingüísticas (conceptos o significados) y la existencia y el contenido de las instituciones jurídico-políticas que podemos crear, y a las que podemos referirnos mediante el uso de las primeras.

Un punto fundamental de la propuesta del PJN es que las instituciones jurídicas presuponen y se construyen mediante instituciones más básicas: instituciones lingüísticas. La idea de que las instituciones jurídico-políticas dependen de, y serían imposibles sin el lenguaje no ha recibido en la teoría jurídica un tratamiento 
equilibrado. En la historia de esta disciplina se ha pasado de concebir el derecho sobre bases metafísicas que ignoran completamente su dependencia lingüística, a entenderlo como una realidad puramente lingüística. En la visión del PJN puede decirse que existe una relación de estratificación entre estos dos tipos de instituciones. Este es un punto que se pueda desarrollar con profundidad aquí. Pero la idea general es que una institución jurídico-política se apoya en, y presupone el lenguaje.

(ii) Un concepto jurídico institucional constituye un ejemplo o caso específico de institución lingüística. Es una institución lingüística que delimita un tipo de institución jurídica y cuyas instancias de aplicación son específicos ejemplos de instituciones jurídicas.

23 Es importante poner énfasis en que, entre los conceptos institucionales, por una parte, y las instituciones jurídicas, políticas o religiosas, por la otra, existe una relación de ejemplificación, que no es una relación inferencial, ni menos aún de identidad. Entre el contenido de un concepto institucional y la existencia de una determinada institución que recae en su ámbito de aplicación hay una relación necesaria o constitutiva, i.e. el concepto establece un conjunto de criterios que todo caso de aplicación necesariamente satisface. No ver la distinción entre las instituciones lingüísticas y aquellas instituciones jurídicas, políticas, religiosas, etc. que con el lenguaje podemos crear e identificar significa no ver la distinción entre diversos tipos de normatividad. Una normatividad semántica o conceptual y una normatividad 'práctica': jurídica, política, religiosa, etc. ${ }^{19}$ No ver con claridad el tipo de relación existente entre el concepto y aquello a lo que se aplica tiene resultados ciertamente contraproducentes. Por una parte, allana el camino a la tesis de la traducibilidad de las normas o razones de un tipo (provenientes de nuestras instituciones lingüísticas) en normas o razones del otro tipo (provenientes de nuestras instituciones jurídicas, políticas, religiosas, etc.). Por otro lado, opaca un hecho importante: aun no siendo traducible en enunciados sobre el contenido de las instituciones que permite identificar, todo concepto institucional delimita en un determinado modo una clase de institución y, consecuentemente, podremos llegar a conclusiones sustanciales muy distintas acerca de la misma según cómo hayamos identificado el concepto. En otras palabras, no ver con claridad la relación entre el contenido del concepto y el de las instituciones (jurídicas, políticas, religiosas, etc.), cuya identificación el concepto hace posible, conduce a no ver con claridad la relevancia sustancial del contenido del concepto al momento de hacer aserciones y valoraciones relativas a las instituciones ya identificadas sobre su base.

Es interesante notar que, una vez que distinguimos el concepto institucional de las instituciones a las que el concepto se aplica, podemos distinguir los enunciados referidos al concepto de los enunciados referidos a tales instituciones. Los primeros son enunciados típicos de una teoría general del derecho, los segundos son enunciados doctrinarios, típicos de la ciencia o la dogmática jurídicas. Extendiendo la distinción propuesta por Raz entre enunciados jurídicos puros (o acerca del contenido de un derecho) y enunciados jurídicos aplicativos (o de acuerdo con un derecho ${ }^{20}$, podemos distinguir entre enunciados conceptuales puros o acerca del contenido de un concepto y enunciados conceptuales aplicativos o de acuerdo con un concepto. En todo caso, tomando nuevamente como ejemplo el concepto de derecho, los enunciados conceptuales puros que identifican o analizan el contenido de este concepto pueden ser determinantes al momento de obtener conclusiones doctrinarias o judiciales en un caso en particular. ${ }^{21}$ En otras palabras, conjugado con premisas adicionales, dicho concepto 
permite obtener conclusiones sustantivas relevantes sobre aquello que es o no derecho en un determinado tiempo y lugar. Sin embargo, no por ello los enunciados conceptuales puros sobre el concepto de derecho son por sí mismos traducibles en enunciados doctrinales o judiciales acerca del contenido de un derecho.

\section{4 Sobre la posibilidad y la relevancia de los enunciados analíticos} metafísico y otro puramente epistémico. ${ }^{23}$ Un enunciado analítico a priori en sentido metafísico es un enunciado cuya verdad es necesaria en virtud de hechos independientes del lenguaje. Un enunciado analítico a priori en sentido epistémico es uno cuya verdad está justificada necesariamente sobre la base de consideraciones exclusivamente relacionadas con su significado. Los datos clave para la admisión de enunciados analíticos a priori en este último sentido están dados por la idea epistémica de justificación y por la tesis según la cual ciertos enunciados deben ser creídos, aceptados o sostenidos como verdaderos, i.e. están epistémicamente justificados, sobre la base de premisas puramente semánticas o conceptuales. En otras palabras, la idea de que consideraciones relativas al significado o a los conceptos (conceptos que, adviértase, sí dependen de datos empíricos extra-lingüísticos) constituyen una justificación suficiente para aceptar o sostener la verdad de otros enunciados.

punto es altamente relevante dado que, si no contamos con una respuesta que bloquee la tesis escéptica general en contra de la posibilidad de enunciados analíticos $a$ priori, el método que propone el PJN debería ser abandonado ab initio. En tal sentido, asumiré que la distinción presentada es factible y que hay una forma no metafísica de entender el carácter a priori de los enunciados que analizan conceptos. ${ }^{24}$ Sobre esta base, podemos advertir que la propuesta del PJN involucra dos cosas. En primer lugar, una investigación de carácter empírica y a posteriori tendiente captar el contenido de los conceptos jurídicos generales efectivamente existentes en una determinada práctica. Es oportuno subrayar que esta indagación sustantiva se refiere (identifica, describe, explica) conceptos y no las específicas instituciones jurídicas a las que los conceptos se aplican. Cosa que sí hacen, por ejemplo, las teorías doctrinarias o sociológicas que se refieren directamente a (identifican, describen, explican) específicas prácticas o instituciones jurídicas. En segundo lugar, involucra también una indagación analítica, a priori, en la medida en que intenta establecer qué presupuestos y qué consecuencias están epistémicamente justificados sobre la base de conceptos identificados. ${ }^{25}$ 
28 Así caracterizado, el discurso analítico del PJN se pone en contraste con aquellos discursos que se refieren directamente al 'mundo' o a 'la realidad' jurídica y obtienen conclusiones sustantivas acerca de cómo ese 'mundo' es, o cómo debe ser. En un sentido estrictamente epistémico, un discurso analítico de conceptos es un discurso 'interno', es decir, que se justifica exclusivamente sobre la base de consideraciones relativas al significado o al contenido de conceptos involucrados. ${ }^{26} \mathrm{Sin}$ embargo, en un sentido práctico, es un discurso propuesto desde un punto de vista 'externo', es decir, que no se compromete con la corrección o justificación de las concretas instituciones a las que los conceptos analizados se aplican, ni con la de las creencias que las tienen en pie. ${ }^{27}$

Solo por mencionar algunos ejemplos de estos enunciados analíticos, según Hans Kelsen, una de las características necesarias de todo derecho es su carácter dinámico. ${ }^{28}$ Podríamos discrepar con este autor sobre si este es o no un rasgo necesario o conceptual del derecho. Sin embargo, ello no impide advertir que, en cualquier caso, enunciados como "Si el derecho es dinámico entonces prevé mecanismos que regulan su propia producción" o bien, "Si una institución es jurídica entonces hay un mecanismo para modificarla" son analíticos y estamos justificados en aceptar su verdad solo sobre la base del significado de "dinámico" en el primer caso, y sobre el concepto de "derecho" en el segundo. Del mismo modo, podemos citar el caso de Herbert Hart quien ha puesto énfasis en que todo derecho se basa en una regla social. ${ }^{29}$ Consecuentemente, un análisis del concepto de derecho en esta perspectiva hartiana nos permitiría afirmar que "Si algo es un sistema jurídico entonces no se apoya en un mero hábito de obediencia”. Un último ejemplo: según Joseph Raz, el derecho necesariamente pretende autoridad y la autoridad ha de entenderse en términos de razones excluyentes..$^{30} \mathrm{En}$ tal sentido, el enunciado "Si algo es una norma jurídica, entonces pretende ser una razón independientemente del contenido" está justificado en virtud del significado de los términos involucrados. Este tipo de afirmaciones - y no aquellas que de hecho identifican el contenido de los conceptos en cuestión -, son analíticas en el sentido epistémico identificado en el punto anterior.

Ciertamente, como cualquier otro tipo de enunciados, los que identifican o analiza conceptos pueden figurar en argumentos que justifican conclusiones de carácter empírico o de carácter práctico. Justamente por ello, dichos enunciados no son inertes y pueden tener relevancia en los estudios dogmáticos o de ética normativa. El que una tesis sea analítica de un concepto y que su justificación sea a priori, no implica que no pueda ser parte en un razonamiento teórico o práctico sobre cuestiones sustantivas empíricas o morales. Sin embargo, sí implica que ella no es sinónima de, ni puede ser traducida en, tesis sustantivas empíricas o morales, y que, a partir de ella, por sí sola, no podemos inferir ninguna conclusión acerca de cómo el mundo efectivamente es, o debe ser.

31 En síntesis, una metateoría jurídica como la del PJN, cuando identifica un concepto jurídico propone en tercera persona enunciados sustantivos cuya verdad o falsedad depende de la evidencia empírica. Concretamente, dependen de la práctica y de la autocomprensión de los agentes involucrados en la misma. Asimismo, cuando analiza un concepto y lo relaciona con otros conceptos establece conexiones estrictamente semánticas, cuya verdad se justifica solo en virtud del contenido de los conceptos identificados. Esta perspectiva entiende los conceptos como un conjunto de propiedades que solo pueden considerarse normativas en un sentido estrictamente semántico. Desde ese punto de vista, el análisis establece aquello que debemos o 
estamos autorizados a aceptar como verdadero sobre la base del contenido de un concepto. Sin embargo, no nos permite obtener directamente conclusiones, acerca de cómo, contingentemente, una institución es, ni acerca de cómo, desde un punto de vista moral, ella debe ser. En todo caso, ello no significa que los conceptos y el análisis propuestos sean irrelevantes, puesto que ellos pueden ser conjugados con información adicional y habilitar inferencias sustantivas muy diferentes acerca de cómo la realidad institucional es o debe ser.

\section{La identificación y análisis de un concepto en concreto}

En este apartado intentaré mostrar en qué sentido las tres propuestas identificadas echan luz sobre cuestiones diferentes y no son excluyentes entre sí. Al mismo tiempo, intentaré poner de relieve en qué sentido, si interpretadas como diferentes modos de entender los conceptos jurídicos, las propuestas de Alf Ross y de Dworkin caen en ciertas dificultades que no afectan al enfoque del PJN.

\section{El delito de brujería}

La creencia (espero falsa) en la existencia de seres sobrenaturales malignos, con poderes mágicos y capaces de producir efectos siniestros ha siempre existido y existe aún en nuestros días. Ahora bien, puede decirse que fue parcialmente esta clase de creencias la que dio lugar a un tipo de institución, paradójicamente moderna, cuya existencia puede fijarse entre finales del 1400 y finales del 1700. Durante estos tres siglos, en diversos estados católicos y protestantes, tribunales eclesiásticos y civiles capturaron, sometieron a proceso, torturas y condenas de distintos tipos a personas (generalmente mujeres) acusadas de un específico tipo de delito: la brujería. Puede decirse que esta institución jurídica nace en 1484 con la bula de Inocencio VIII en la que la brujería se considera una específica forma de herejía y se autoriza a dos padres dominicanos alemanes (Heinrich Institor Kramer y Jakob Sprenger) a reglamentar el modo de proceder para detectar y eliminar este tipo de ofensa a los valores del orden, la fe y la iglesia.

El tratado que estos sacerdotes escribieron, el Malleus Maleficarum, tuvo 34 reediciones y más de 35.000 copias impresas. ${ }^{31}$ Puede considerarse un manual y, a la vez, un código de brujería que, durante casi toda la historia de la institución, sirvió como guía a las autoridades jurisdiccionales, religiosas y seculares que aplicaron el delito de brujería. En tal sentido, el Malleus Maleficarum es un claro ejemplo de lo que la teoría jurídica de corte realista subraya cuando sostiene el carácter creativo, y de fuente de derecho, de la doctrina jurídica. En efecto, esta obra propone una detallada reflexión acerca de la naturaleza de la brujería, pero también un conjunto de normas concretas que los jueces debían aplicar a aquellas personas que incurrían en este tipo de herejía. Así, por una parte, el texto identifica una serie de hechos que permiten determinar si una persona es, o no, una bruja, y, por otra parte, una serie de consecuencias deónticas aplicables sobre la base de esta constatación. Contemporáneamente, además de las normas sustantivas sobre la brujería, el libro establece reglas sobre el juez competente, distingue diversas etapas del proceso por bujería según sea el tipo de caso y, concretamente, delimita trece modos en los que puede culminar esta clase de proceso. 
Lo que me interesa destacar en este contexto es que, conforme a lo que he sostenido hasta aquí, teniendo en cuenta la práctica de esta institución sería posible identificar 'el concepto' de brujería en los tres sentidos señalados hasta aquí: en el de Ross, en el de Dworkin, y en el que propone el PJN.

\section{1 La aplicación de la perspectiva de Ross}

Aplicando las tesis de Alf Ross, para identificar el concepto de brujería deberíamos proceder como con cualquier otra noción institucional. En primer lugar, deberíamos decir que ciertamente las brujas (entendidas como seres naturales o sobrenaturales) no existen. Quien cree en semejante cosa tiene una creencia mágica que contradice e ignora lo que nos enseña la ciencia. Ahora bien, según este enfoque, tampoco cabe admitir la existencia de la brujería como una realidad institucional. Admitir este tipo de entidades institucionales sería un resabio de aquellas creencias mágicas, científicamente injustificadas. En suma, la creencia de que el concepto institucional de bruja se refiere a algo que se ejemplifica o instancia en el mundo es también sistemáticamente falsa puesto que se basa en la admisión de entidades que no existen. Al respecto, como vimos, el análisis de Ross sugiere reducir el concepto y la realidad institucional de la brujería al conjunto de reglas que se aplicaron en un tiempo y lugar determinados. En esta perspectiva, decir que $\mathrm{S}$ es una bruja significa que $\mathrm{S}$ satisface ciertas condiciones y, justamente por ello, se siguen ciertas consecuencias deónticas. Concretamente, según el Malleus Maleficarum, si una persona S:

a- Es gravemente sospechada de haber realizado actos invocando al diablo y, después del interrogatorio, se niega a confesar y abjurar o, habiendo abjurado, reincide en dichos actos.

b- Es violentamente sospechada de haber realizado actos invocando al diablo, y después del interrogatorio no abjura.

c- Es denunciada por haber realizado actos por influencia del diablo y es contumaz o fugitiva.

d- Es capturada en flagrancia en la comisión de un acto bajo la influencia del diablo.

e- Confiesa haber realizado actos por influencia del diablo, y no se arrepiente.

f-Confiesa haber realizado actos por influencia del diablo, se arrepiente, y luego reincide.

g- Es denunciada y existe prueba, mediante legítimos testigos, de que ha realizado actos bajo la influencia del diablo.

$S$ es una bruja y, consecuentemente,

h-Debe ser sometida a purificación.

i-Debe ser sometida a interrogatorio (tortura).

$\mathrm{j}$-Debe ser excomulgada, $\mathrm{y}$, en su caso, privada de sus fueros.

k-Puede ser, a discreción del juez, condenada a estar en pie en las puertas de una iglesia, o a hacer determinadas peregrinaciones.

1-Debe ser sometida a cárcel perpetua, a pan y agua,

m-Debe ser enviada al brazo secular (ejecutada en la hoguera, en una plaza pública, fuera de la iglesia, en un día no festivo). ${ }^{32}$

La forma en que Ross entiende este tipo de conceptos jurídicos da pie a algunas consideraciones críticas. Concretamente, siguiendo la estrategia de Ross una bruja es alguien que satisface alguna de las condiciones enumeradas (en el conjunto a-g) y a la 
cual le son aplicables las consecuencias previstas (en el conjunto h-m). En este sentido, como vimos, esta propuesta hace colapsar la distinción entre el concepto institucional general de bruja o brujería y el contingente contenido que un ejemplo de esta institución tiene en un momento y lugar específicos. Sin embargo, estas dos cosas son diferentes y es conveniente separarlas. De hecho, es posible que en un grupo social exista un ejemplo de un tipo de institución, sin que dicho grupo disponga del concepto general correspondiente..$^{33}$ Por supuesto, esto es imposible si asumimos la propuesta de Ross en la que el concepto no es otra cosa que el contenido de una específica institución vigente en un lugar y tiempo determinados. A su vez, al hacer esta equiparación se oculta el hecho de que es el concepto general el que permite identificar la existencia de ejemplos de instituciones del mismo tipo.

En segundo lugar, en su trabajo de 1951, Ross asocia la aceptación de entidades, propiedades o hechos institucionales a la aceptación de entidades, propiedades, o hechos meramente aparentes, generados por supersticiones primitivas. Concretamente, Alf Ross compara nuestras creencias de que bajo ciertas circunstancias existen ciertos hechos o propiedades institucionales con las creencias de los integrantes de una tribu primitiva en que, bajo ciertas circunstancias, un individuo deviene tû-tû. ${ }^{34}$ Al hacer esta analogía, asimila la aceptación y la justificación de enunciados referidos a entidades o propiedades institucionales, que pueden perfectamente explicarse sobre bases empíricas compatibles con nuestro conocimiento científico, con la aceptación y la justificación de enunciados referidos a entidades de carácter mágico, cuya admisión puede considerase sistemáticamente falsa, y que deberíamos rechazar. En contraste con lo que Ross sugiere, conforme al PJN, las instituciones jurídico-políticas son parte de una 'realidad social' cuya existencia efectivamente depende de las creencias y actitudes de los miembros de un grupo social. Ahora bien, el hecho de que estas creencias sean verdaderas o falsas, o que las actitudes de los miembros del grupo sean moralmente meritorias o no, son datos irrelevantes a los fines de la existencia e identidad de una determinada institución, ya que estas dependen de los contenidos de tales creencias y actitudes, pero no de la verdad o del mérito moral de las mismas. En la literatura sobre el tema se encuentran posiciones contrastantes con respecto a la relevancia de la verdad o falsedad de las creencias constitutivas de las instituciones sociales. Según Searle, el hecho de que ciertas instituciones se basen en creencias falsas puede hacerlas aún más sólidas y duraderas. ${ }^{35}$ Sin embargo, es posible conjeturar que las instituciones basadas en creencias falsas, como ciertos tipos de esclavitud, el delito de brujería o la monarquía de origen divino, por citar algunos ejemplos, irán desapareciendo a medida en que se demuestre la falsedad de las creencias en las que ellas se basan.

En todo caso, una vez que aceptamos la existencia de una realidad social, no natural, es importante no confundir la admisión, por ejemplo, de la existencia de la institución de 'la brujería' y la de objetos institucionales como 'las brujas' (en la medida en que se satisfacen las condiciones previstas por las normas jurídicas correspondientes), con la admisión de la existencia de un hecho natural o sobrenatural como sería la existencia de seres que en virtud de un pacto con el diablo comen a los niños, provocan pestes, hambrunas y otros portentos. La teoría de la realidad institucional intenta subrayar que hay buenas razones para aceptar lo primero, pero en ningún caso sugiere que haya razones para aceptar lo segundo.

Teniendo en cuenta estas consideraciones, puede decirse que las tesis de Ross se sustentan en algunos razonamientos implícitos falaces. En primer lugar, como vimos, 
su propuesta se apoya en una analogía impropia. Concretamente, teniendo en cuenta algunas similitudes entre creencias mágicas y creencias en instituciones normativas, sugiere que, así como debemos rechazar las primeras, debemos también rechazar las segundas. Sin embargo, existen algunas diferencias muy relevantes entre estos dos tipos de creencias. Por mencionar una fundamental: las segundas, y no las primeras, pueden estar racionalmente justificadas en términos compatibles con el conocimiento científico. De hecho, a partir de ciertas creencias y preferencias morales, políticas, económicas, etc. podemos intencionalmente diseñar y aceptar, por ejemplo, un determinado órgano de control constitucional o un nuevo tipo de sociedades comerciales. En tal caso, la 'existencia' de este tipo de estado o de sociedades comerciales no se basa en ningún tipo de creencia supersticiosa o de tabú. En tal sentido, establecer una analogía entre la admisión de una realidad mágica y la de una realidad institucional es altamente engañoso. Si la aceptásemos no estaríamos en condiciones de comprender, por ejemplo, la inmensa cantidad de esfuerzo epistémico que se dedica a la segunda en comparación con el dedicado a la primera. En otras palabras, comprender la existencia de múltiples teorías que indagan sobre los procesos mediante los cuales la realidad institucional emerge, se afianza y se destruye. En segundo lugar, a partir de esta analogía impropia, Ross hace una generalización impropia o, si se quiere, comete una falacia de composición. A partir del análisis de una institución como tû-tû de la tribu Noit-cif, que se basa en creencias sistemáticamente falsas - y que en esto es similar a la brujería de los siglos XV y XVIII - sugiere que todas las instituciones son del mismo tipo. Nuevamente, apreciar el contraste entre instituciones como tû-tû o la brujería y otras como el control de constitucionalidad, las sociedades comerciales, o la pensión por invalidez, nos permite tomar conciencia de que, al igual que cualquier otra construcción humana, las instituciones sociales no son todas del mismo tipo. Algunas pueden estar basadas en ideas místicas y, como afirma Ross respecto de tû-tû, producir miedo y terror. ${ }^{36}$ Otras, en cambio, pueden estar fundadas en creencias ilustradas y producir efectos de signo exactamente contrario.

41 Ciertamente, las críticas que acabo de mencionar a la posición de Ross podrían fácilmente evitarse. Pero, para ello, es necesario abandonar la idea de que los dichos de este autor ofrecen una estrategia para la identificación de un concepto jurídico y entenderlos como la propuesta de un método para describir o hacer explícito el contenido normativo de una específica institución.

\section{2 La aplicación de la perspectiva de Dworkin}

En la perspectiva de Dworkin, el teórico que intenta identificar el concepto de bruja o brujería tiene que concentrarse, no ya en un específico ejemplo, sino en distintos ejemplos de esta clase de institución para proponer, en primera persona, un conjunto de principios que justifiquen la mejor versión que de ellos pueda ofrecer. Como sabemos, conforme a la visión metodológica de este autor, y en contra de la visión del positivismo realista, es plausible admitir la existencia de instituciones, hechos, propiedades normativas, $\mathrm{y}$, ciertamente, todos ellos han de considerarse constructos sociales. Es decir, Dworkin y el PJN coinciden sobre este punto. Ahora bien, en contraste con este último, según Dworkin, respecto de 'la realidad social' no es posible adoptar un punto de vista 'externo', en un sentido práctico. ${ }^{37}$ Identificar el concepto de brujería implica ofrecer una teoría normativa que, en alguna medida, la justifica. 
Consecuentemente, y en la misma medida, implica comprometerse con los valores que ese tipo de institución encarna. ${ }^{38}$

Un ejercicio dworkiniano respecto del delito de brujería podemos encontrarlo en la primera parte del manual Malleus Maleficarum donde los autores se presentan como intentando ofrecer "la sana comprensión del Cánon" y, así, al afrontar los desacuerdos existentes al respecto, sostienen que el modo correcto de entenderlo es como una forma de herejía que en última instancia representa una "ofensa a la majestad divina". En otros pasos del texto afirman que el valor que está en cuestión es la sacralidad de la Iglesia y también el orden natural. En todo caso, no cabe duda de que las lecturas que ofrecen responden al objetivo de justificar esta clase de institución y que están realizando el mismo tipo de ejercicio interpretativo que propone Dworkin cuando discute sobre si la cortesía es una forma de respeto a las personas, o si el derecho es o no una forma de hacer respetar derechos morales individuales. Ejercicio que normalmente los juristas hacen y que podemos considerar de alto interés.

Ahora bien, respecto de la propuesta de Dworkin es posible realizar las siguientes consideraciones críticas. En primer lugar, tal como el ejemplo tomado del Malleus Maleficarum muestra, el ofrecimiento en primera persona de una teoría que hace inteligible a la brujería no es un obstáculo para, paralelamente, identificar un concepto criterial, que, conforme a las creencias de los participantes en la práctica, delimita a este tipo de institución. En efecto, Kramer y Sprenger hacen ambas cosas. Por una parte, sostienen que los valores que justifican la persecución del delito de brujería son la majestad divina, la sacralidad de la iglesia y el orden natural. A su vez, se detienen con cierta profundidad en tratar de mostrar cuáles son sus características distintivas. En opinión de estos autores, tal como la entiende la comunidad relevante, (i) la brujería supone necesariamente un pacto con el diablo. Asimismo, dado que todos admiten que el diablo no puede operar causalmente sobre los cuerpos materiales, se sigue que (ii) la obra de brujería es causalmente atribuible a la bruja, y no al diablo. Por otra parte, teniendo en cuenta que el diablo no puede operar a menos que alguien voluntariamente se preste a llevar a cabo sus propósitos, se sigue también que (iii) la responsabilidad de la acción de brujería es imputable a la bruja, y no al diablo. Por último, relevan que conforme a la doctrina los efectos producidos mediante la brujería no son efectos ficticios o fantásticos, como los que dan lugar a otros tipos de herejías, sino efectos empíricos, reales. En otras palabras, (iv) un acto de brujería produce siempre un cambio en el mundo.

Es relevante subrayar que, aun cuando un concepto criterial pueda considerarse fruto de un acuerdo o convención de los participantes en una práctica, su identificación no lo es. Es posible errar en la identificación del conjunto de criterios que, según la práctica, definen una institución. La tarea de identificación del concepto criterial de brujería es claramente sustantiva, en la medida en que intenta captar las características que la brujería efectivamente tiene a los ojos de los participantes. Asimismo, es controvertible, como lo muestra el debate presente en la obra de Kramer y Sprenger, en la que explícitamente se presenta la opinión de quienes entienden que es posible que las brujas actúen sin intervención del diablo, o que sean directamente víctimas de él y no responsables de sus acciones, o que los efectos que causan puedan ser solo ficticios y no reales.

En suma, así como lo ha mostrado Joseph Raz respecto del concepto de derecho ${ }^{39}$, es posible sostener que, respecto de las instituciones sociales en general, nada obsta a que, 
a la par de un concepto 'interpretativo', podamos identificar un concepto criterial. Consecuentemente, no parece justificado aceptar que los conceptos aplicables a instituciones sociales tengan que ser necesariamente concebidos como conceptos 'interpretativos', en el sentido que Dworkin confiere a este término. A diferencia de un concepto interpretativo, el concepto criterial nada dice sobre el valor moral de un determinado conjunto de normas (i.e. una institución), ni sobre las condiciones de verdad de las proposiciones que las identifican y las aplican. A pesar de esto, un concepto criterial es siempre relevante desde un punto de vista práctico, y no solo en una perspectiva sociológica descriptiva, o guiada por un interés meramente taxonómico. Por ejemplo, si adoptamos el concepto criterial identificado por Kramer y Sprenger, una persona que reivindica públicamente la existencia del diablo y la conveniencia de aliarse con él, pero que no ha dado lugar a ningún resultado material que pueda ser entendido como intermediado por el diablo, conforme al requisito (iv) de la definición de estos autores no podría ser considerada una bruja. Quizás pueda ser considerada como autora de otro tipo de herejía, o imputada por apología de la brujería, pero no por ser una bruja. Esta conclusión puede ser determinante en una discusión doctrinal, jurisprudencial o moral respecto de la calificación normativa de una acción y está parcialmente justificada sobre la base del concepto criterial de bruja identificado anteriormente. Esto desmiente la tesis de la irrelevancia práctica del concepto entendido en este sentido.

Por otra parte, el ejemplo de la brujería permite hacer explícito un argumento, no menor, en contra de una concepción interpretativa de los conceptos jurídicos. Instituciones como la brujería se basan en creencias sistemáticamente falsas de los participantes que la justifican. En este tipo de casos, por hipótesis, el teórico tiene razones - necesariamente 'externas' respecto de las creencias de los participantes para sostener que la institución en cuestión carece de una justificación apropiada. Dworkin ciertamente admite que este tipo de situaciones pueden verificarse. Sin embargo, la respuesta que emerge de su posición es desconcertante.

Aplicando el enfoque de este autor, un estudioso que se refiere a instituciones sociales se encuentra ineludiblemente en una discusión interpretativa y toma decisiones que lo comprometen moralmente, en alguna medida, con alguna lectura justificativa respecto de las mismas. Ahora bien, si esto es así, el teórico no puede coherentemente - i.e. sin caer en contradicción pragmática - afirmar que todas las teorías susceptibles de justificar el tipo de institución al que se refiere se basan en creencias falsas o, lo que es lo mismo, que ninguna permite justificarla adecuadamente. Esto debido a que tener una lectura interpretativa y participar en un debate sobre un concepto interpretativo no consiste simplemente en aceptar valores o tener una actitud moralmente comprometida en abstracto; es ofrecer en primera persona una teoría que, en alguna medida, hace inteligible o justifica el tipo de institución sobre la que se discute. En otras palabras, es hacer algo (ofrecer razones que hacen inteligible o justifican un tipo de institución) que, en casos como el señalado, entra en contraste con lo que decimos (que no existen razones que hagan inteligible o justifiquen ese tipo de institución). Consecuentemente, en esta clase de casos referidos a instituciones basadas en creencias sistemáticamente falsas, la propuesta de Dworkin puede ser reducida al absurdo: asumirla conduce inevitablemente a una contradicción pragmática. Ello porque no es posible adoptar una actitud interpretativa respecto de un tipo de institución $y$, 
contemporáneamente, sostener que ella carece de toda posible justificación. Lo cual, en un tipo de caso como el analizado, es lo que el teórico tiene razones para sostener.

Las consideraciones presentadas no pretenden negar el interés que puede tener la identificación de aquellos principios que, en opinión de quien los propone, brindan la mejor justificación de un tipo de institución. En otras palabras, no pretenden negar el interés de una teoría interpretativa como la que Dworkin propone. Lo que pretenden mostrar es que no parece adecuado concebir esta propuesta como una empresa de carácter conceptual. La identificación de un concepto interpretativo tal como lo entiende Dworkin no compite con, ni sustituye, la identificación de un concepto criterial. Asimismo, identificar un concepto criterial, que delimita un tipo de institución social, no implica ni requiere asumir una teoría interpretativa, i.e. no implica ni requiere contar con un 'concepto' interpretativo tal como los entiende Dworkin. Ahora bien, en contraste con esta propuesta, si asumimos que la teoría interpretativa de Dworkin es efectivamente una forma de entender e identificar los conceptos institucionales, cabe concluir que la propuesta del PJN se encuentra en una mejor posición. Por una parte, la identificación y análisis conceptual que propone el PJN no quita sentido ni resta importancia al ofrecimiento de teorías justificativas respecto de cualquier institución. Tarea que es independiente de, y compatible con, el análisis que le PJN propone. En tal sentido, esta posición no prescribe metodológicamente abstenerse de participar en debates de filosofía política o moral como los que propone Dworkin, solo que no entiende que tales debates sean un ejemplo de identificación y análisis conceptual. Por otra parte, el método del PJN permite distinguir y expresar algo que la teoría de Dworkin no puede distinguir ni expresar. Concretamente, la perspectiva del PJN permite identificar ejemplos de un tipo de institución sin que ello implique un juicio justificativo. De este modo, permite, sin caer en contradicción, identificar una institución y contemporáneamente sostener que no disponemos con relación a ella de ninguna teoría adecuada, inteligible, o verdadera, capaz de justificarla. Esta posibilidad, vedada a la teoría de Dworkin, supone colocarse en un sentido práctico o valorativo - en un punto de vista externo respecto de las creencias que sostienen y justifican el tipo de institución en cuestión. Ciertamente, un punto de vista externo respecto de una institución no es un punto de vista arquimediano o neutral en términos absolutos. Sencillamente, como se acaba de indicar, es externo respecto de la creencias y actitudes de los aceptantes de la institución en cuestión.

\section{Consideraciones finales}

50 A lo largo del trabajo me he referido a tres posiciones que se presentan como distintos modos de entender, identificar y analizar una cierta clase de conceptos jurídicos. Al respecto, he mostrado que si efectivamente lo fuesen, las tesis de Alf Ross y Ronald Dworkin, que se proponen como fuertemente críticas y excluyentes respecto del enfoque del PJN, merecerían ser objetadas en virtud de las dificultades que generan. Ahora bien, puede decirse que, para cada una de estas posiciones, los conceptos jurídicos a los que se refiere son conjuntos de reglas o principios de diferente tipo: reglas semánticas, según el PJN, reglas de un específico sistema jurídico, según la propuesta realista de Alf Ross, y principios político-morales, según el enfoque interpretativista de Ronald Dworkin. Conforme he tratado de mostrar, identificar cada 
una de estas clases de normas constituye un tipo diferente de empresa. Sobre esta base, la sugerencia es que no parece apropiado entender a estas posiciones como involucradas en el mismo tipo de operación: la identificación y análisis de conceptos jurídicos. En realidad, la primera efectivamente conduce a identificar el contenido de un concepto general, la segunda, el contenido de una institución jurídica vigente en un determinado tiempo y lugar, la tercera, el contenido de una teoría justificativa de un tipo de institución. En otras palabras, las tres posiciones se refieren a objetos distintos y están guiadas por objetivos diferentes. En tal sentido, no son competitivas entre sí.

\section{BIBLIOGRAFÍA}

Arriagada Caceres, M.B. (2018). Tres problemas de Atria sobre los conceptos jurídicos ilustrados en el concepto de derecho subjetivo. Revista chilena de derecho, 45(1), 7-31.

Atria, F. (2016). La forma del derecho. Madrid, Spain: Marcial Pons.

Barberis, M. (2004). Una breve storia della filosofia del diritto. Bologna, Italy: Il Mulino.

Bix, B. (1993). Law, Language, and Legal Determinacy. Oxford, England: Clarendon Press.

Boghossian, P.A. (2008). Content and Justification. Philosophical Papers. Oxford, England: Oxford University Press.

Dworkin, R. (1986). Law's Empire. London, England: Fontana Press.

Dworkin, R. (1996). Objectivity and Truth: You'd Better Believe it. Philosophy and Public Affairs, 25(2), 87-139.

Dworkin, R. (2006). Justice in Robes. Cambridge, Mass.: Harvard University Press.

Hart, H.L.A. (1994) The Concept of Law. Oxford, England: Clarendon Press (Original work published 1961)

Iglesias, M. (1999). El problema de la discreción judicial. Una aproximación al conocimiento jurídico. Madrid, Spain: Centro de Estudios Políticos y Constitucionales.

Kelsen, H. (1979). Teoría pura del derecho (R.J. Vernengo, Trans.). Ciudad de México, Mexico: UNAM. (Original work published 1960)

Kramer, H.I. \& Sprenger, J. (2003). Il Martello delle Streghe. La sessualità femminile nel "transfert" degli inquisitori (A. Verdiglione, Trans.). Milano, Italy: Spirali. (Original work published 1486-1487).

Lifante Vidal, I. (1999). La interpretación jurídica en la teoría del derecho contemporánea. Madrid, Spain: Centro de Estudios Políticos y Constitucionales.

MacCormick, N. (1998). Norms, Institutions and Institutional Facts. Law and Philosophy, 17, 310-345.

Mindus, P. (2009). A Real Mind. The Life and Work of Axel Hägerström. Dordrecht, The Netherlands: Springer. 
Narváez, M. (2002-2003). Enunciados filosóficos vs. enunciados teóricos. El caso de la textura abierta del derecho. Analisi e diritto 2002-2003, pp. 211-240.

Nino, C.S. (1984). Introducción al análisis del derecho (2 $2^{\text {nd }}$ ed.). Buenos Aires, Argentina: Astrea. Quine, W.V.O (1961). From A Logical Point of View (2 ${ }^{\text {nd }}$ ed.). New York, NY: Harper \& Row. (Original work published 1953)

Raz, J. (1970). The Concept of a Legal System, Oxford, England: Oxford University Press.

Raz, J. (1979). The Authority of Law. Oxford, England: Oxford University Press.

Raz, J. (1998). Two Views of the Nature of The Theory of Law. Legal Theory, 4, 249-282.

Raz, J. (2009). Can There be a Theory of Law. In Between Authority and Interpretation (pp. 17-46).

Oxford, England: Oxford University Press.

Redondo, M. C. (2018). "El método y el objeto de la teoría jurídica. La ambigüedad internoexterno”. Análisis Filosófico, XXXVIII(2), 115-156.

Ross, A. (1957). Tû-tû. Harvard Law Review, 70(5), 812-825. (Original work published 1951)

Ross, A. (1994). Sobre el derecho y la justicia (G.R. Carrió, Trans.) (5a. ed). Buenos Aires, Argentina: Eudeba. (Original work published 1958)

Roversi, C. (2012). Costituire. Uno studio di onlogia giuridica. Torino, Italy: Giappichelli.

Searle, J. (1995). The Construction of Social Reality. London, England: Penguin Books.

Searle, J. (2010). Making the Social World. The Structure of Human Civilization. Oxford, England:

Oxford University Press.

Winch, P. (1990). The Idea of a Social Science and its Relation to Philosophy ( $2^{\text {nd }}$ ed.). London, England: Routledge. (Original work published 1958)

\section{NOTAS}

1. En este contexto las instituciones pueden ser entendidas como conjuntos de reglas che dependen de la aceptación de un grupo social. Cf. Searle 1995: 27-29. O bien, en modo similar, como un conjunto de reglas correspondiente a una práctica social. Cf. MacCormick 1998: 306 ss. Como se verá a lo largo del trabajo, es oportuno mantener una clara distinción entre instituciones o reglas jurídicas e instituciones o reglas lingüísticas/semánticas.

2. Dos precisiones merecen ser realizadas. En primer lugar, al hablar del PJN me refiero a aquel tipo de positivismo jurídico que no reduce el derecho a datos puramente empíricos, sino que lo concibe como un conjunto de contenidos normativos. En segundo lugar, cabe subrayar que no todos los autores que asumen esta posición sostienen exactamente las mismas tesis respecto de los conceptos jurídicos. Por mencionar un ejemplo, autores como Joseph Raz, Herbert Hart y Eugenio Bulygin sostienen posiciones distintas sobre los conceptos jurídicos, pero todas ellas contrastantes respecto de las propuestas por Dworkin y Ross. En tal sentido, lo que diré en este trabajo vale en general para estas distintas posiciones positivistas y normativistas, aun cuando ellas discrepen entre sí sobre algunas específicas tesis relativas a los conceptos jurídicos.

3. Cf. Dworkin 2006: 9-12. Dworkin 2011: 158-163.

4. Al respecto véase, por ejemplo, Iglesias 1999: 136-141.

5. Cf. Dworkin 1986: 87 y Dworkin 2006: 12.

6. Cf. Dworkin 2011: 161.

7. El ejemplo sobre el derecho Nazi puede verse en Dworkin 1986: 104-108. 
8. Cf. Dworkin 1986: 108.

9. Al respecto, puede consultarse el análisis propuesto en Lifante Vidal 1999: 284-296.

10. Cf. Dworkin 1986: 90.

11. Cf. Dworkin, 2006: 9-21.

12. Cf. Dworkin 2006: 19-20.

13. Cf. Dworkin 2006: 226-240.

14. Cf. Dworkin 2006: 235-240.

15. Cf. Ross 1957: 818. Cabe subrayar que no estoy tratando de identificar una teoría general de los conceptos jurídicos en el pensamiento de Alf Ross. Me estoy refiriendo a sus tesis sobre los conceptos 'tû-tû' que el autor analiza en su famoso ensayo de 1951 y que constituyen una continuación de la crítica a los conceptos jurídicos ya iniciada, en el contexto escandinavo, por Hägerstöm. El enfoque de Ross - que, como el de Olivecrona, no es puramente crítico, sino también constructivo - se propone en la literatura jurídica como ejemplo paradigmático de un enfoque realista y reduccionista de los conceptos institucionales. Véase, por ejemplo, el capítulo de Nino con respecto a "los conceptos básicos del derecho" en Nino 1984: 209-216. También Roversi 2012: 103-118. Respecto de la propuesta inicial de Hägerström, véase Mindus 2009: 157. También Barberis 2004: 118, 122.

16. Cf. Ross 1957: 819-821.

17. Cf. Ross 1958/1994: 168.

18. Ross 1958/1994: 39.

19. Esta falta de distinción conduce a otro tipo de reducción igualmente generadora de confusiones. Me refiero a la idea según la cual un estudio sobre del seguimiento de reglas lingüísticas (como el que ofrece Wittgenstein, por ejemplo) sería directamente trasladable al análisis del seguimiento de reglas jurídicas, o suficiente para la comprensión de estas últimas. Al respecto, véase Bix 1993: 36-62.

20. Raz 1970: 49-50 y Raz 1979: 62.

21. A modo de ilustración, es interesante el ejemplo propuesto por Atria en el que se ven las conclusiones sustanciales a las que se puede llegar respecto de un caso en particular cuando se adopta el concepto de derecho propuesto por Herbert Hart. Cf. Atria 2016: 66-72. Este autor, sin embargo, confunde el hecho de que el concepto puede entrar en un razonamiento práctico y contribuir a determinar su conclusión (idea aplicable al concepto de Hart como a cualquier otro concepto) con la tesis de que el contenido del concepto está ya comprometido y permite por sí solo inferir enunciados sobre contenido de la institución (idea aplicable a los conceptos interpretativos de Dworkin, pero no a los conceptos criteriales tal como los entiende el PJN). Una crítica al análisis de Atria puede verse en Arriagada Cáceres 2018: 7-31.

22. Quine 1953/1961: 20-37.

23. Boghossian 2008: cap. 9.

24. Una forma diferente de entender la distinción entre discursos a priori y a posteriori, pero compatible con la propuesta del PJN puede verse en Narváez 2002-2003: 211-223.

25. Puede decirse que el interés práctico del análisis metateórico depende de la verdad de los enunciados que identifican los conceptos analizados. Es decir, parecería poco útil el análisis de conceptos que no captan, o captan incorrectamente, aquellos efectivamente usados en un tiempo y lugar determinados. Sin embargo, en un sentido epistémico, una indagación puede ser inobjetable aun cuando se llegue a la conclusión de que es escasamente útil porque se apoya en premisas falsas o porque, intencionalmente, analiza conceptos no usados en ninguna práctica.

26. Utilizo aquí la noción de discurso interno en el sentido en que la emplea Peter Winch cuando sostiene que este tipo de discurso no se refiere a eventos empíricos sino a contenidos de significado. Cf. Winch 1958/1990: 111-120.

27. Con respecto a estos dos sentidos las expresiones "punto de vista interno" y "punto de vista externo" véase Redondo 2018: 122-23. 
28. Kelsen 1960/1979: 205.

29. Hart 1961/1994: 51-60.

30. Raz 1979: 28-34.

31. Kramer \& Sprenger 1486-1487 (Se cita la traducción italiana de 2003).

32. Cf. Kramer \& Sprenger 2003.

33. Véase Raz 2009: 37-41.

34. Cf. Ross 1957: 812-813.

35. Searle 1995: 118. Searle 2010: 107-8.

36. Cf. Ross 1957: 812.

37. Dworkin 1986: 76-86. Dworkin 1996: 87-139.

38. Cabe subrayar que una teoría interpretativa-normativa podría no tener necesariamente (como presupone Dworkin) el objetivo de hacer inteligible o identificar los valores que se instancian en determinado tipo de institución. En realidad, podría tener un objetivo exactamente opuesto: quitar inteligibilidad y destacar los defectos que un tipo de institución encarna. En este último caso, sería una teoría claramente normativa y comprometida con valores, pero no comprometida con el tipo de institución a la que se refiere.

39. Cf. Raz 1998: 249-282.

\section{RESÚMENES}

Este trabajo propone una reflexión sobre un tipo de conceptos jurídicos: aquellos que delimitan y permiten identificar instituciones jurídicas. En la primera parte se toman en consideración tres propuestas que se presentan como visiones competitivas sobre el modo de entender este tipo de conceptos institucionales. En primer lugar, la concepción interpretativo-justificativa de Ronald Dworkin, en segundo término, la concepción reduccionista asociada a la teoría realista de Alf Ross. Por último, la concepción criterial sostenida por un positivismo jurídico de tipo normativista. En la segunda parte del trabajo estas tres propuestas se ponen en juego con relación a una institución jurídica concreta: el delito de brujería. Los objetivos del trabajo son fundamentalmente dos. Por una parte, mostrar que el enfoque analítico y metateórico del positivismo jurídico normativista no es reducible a ninguno de los otros dos $\mathrm{y}$, a su vez, relevante desde un punto de vista práctico. Por otra parte, mostrar que la presentación de las propuestas consideradas como enfoques competitivos sobre los conceptos institucionales es engañosa. En realidad, ellas no hablan de los mismo. Están guiadas por objetivos metodológicos distintos y, sobre todo, responden a preguntas diferentes con relación a las instituciones jurídicas.

This paper proposes a reflection on a specific kind of legal concepts: those that delimit and allow to identify legal institutions. In the first part, three proposals, which are presented as competitive visions on this kind of institutional concepts, are taken into account. In the first place, Ronald Dworkin's interpretative-justifying conception, secondly, the reductionist conception associated with the realistic theory of Alf Ross. Finally, the criterial conception supported by a normativist legal positivism. In the second part of the work, these three proposals are put into play about a specific legal institution: the crime of witchcraft. The objectives of the paper are fundamentally two. On the one hand, to show that the analytical and metatheoretical approach of normativist legal positivism is not reducible to any of the other two and, in turn, relevant from a practical point of view. On the other hand, to show that the presentation of the 
considered proposals as if they were competitive approaches to institutional concepts is misleading. They don't talk about the same thing. They are guided by different methodological objectives and, above all, answer different questions concerning legal institutions.

\section{ÍNDICE}

Keywords: institutional concepts, institutions, interpretative concepts, criterial concepts, witchcraft, false beliefs

Palabras claves: conceptos institucionales, instituciones, conceptos interpretativos, conceptos criteriales, brujería, falsas creencias

\section{AUTOR}

\section{MARÍA CRISTINA REDONDO}

Profesora de Filosofía del Derecho, Instituto Tarello para la Filosofía del Derecho Address: Tarello Institute for Legal Philosophy

Via Balbi 30/18

Genoa 16126

Italia

E-mail: cristina.redondo@unige.it 